\title{
Synthèse
}

\section{Eco-intensification dans les montagnes du Vietnam Contraintes à l'adoption de la culture sur couvertures végétales}

François Affholder 1,2,3

Damien Jourdain $2,4,5$

Marion Morize 4,6,7

Dang Dinh Quang ${ }^{8}$

Aymeric Ricome ${ }^{4,9}$

${ }^{1}$ Centre de coopération internationale en recherche agronomique

pour le développement (Cirad),

Unité mixte de recherche (UMR) System,

Hanoï

Vietnam

<affholder@cirad.fr>

${ }^{2}$ International Rice Research Institute (IRRI), Los Baños,

Laguna,

Philippines

${ }^{3}$ Cirad,

UMR Śystem

SupAgro Bât. 27,

2, place Viala,

34060 Montpellier cedex 1

France

<affholder@cirad.fr>

${ }^{4}$ Cirad, UMR G-eau,

34398 Montpellier

<damien.jourdain@cirad.fr>

${ }^{5}$ Cirad,

35, Dien Bien Phu,

Hanoi,

Vietnam

<damien.jourdain@cirad.fr>

6 IRC SupAgro

34093 Montpellier

<marion.morize@yahoo.fr>

7 UPG, Ancienne Chambre d'agriculture, Destrellan,

97122 Baie-Mahault

<marion.morize@yahoo.fr>

${ }^{8}$ Vietnam Academy of Agricultural

Science (VAAS)

Northern Mountainous Agriculture and

Forestry Science Institute (NOMAFSI)

Phu Ho,

Tinh Phu Tho,

Vietnam

<quangdd60@yahoo.com>

9 École d'ingénieurs de Purpan,

Université Toulouse 1

LEREPS

75, voie du TOEC

31076 Toulouse cedex 3

<aymeric.ricome@purpan.fr>

Tirés à part : F. Affholder

\begin{abstract}
Résumé
D'importants efforts sont menés dans le monde tropical pour mettre au point et promouvoir des systèmes de culture sous couverture végétale (SCV) en vue de réduire l'érosion des sols et d'améliorer les bilans minéraux et hydriques. En agriculture familiale, l'adoption des SCV reste cependant limitée par les profonds changements que ces systèmes induisent dans la gestion des ressources de l'exploitation. L'objectif de cette étude menée au Vietnam est d'évaluer la faisabilité, pour différents types d'exploitations, de SCV préalablement mis au point à l'échelle de la parcelle. La méthode repose sur la simulation de ménages rationnels optimisant l'usage des ressources en vue d'objectifs de sécurité alimentaire et de revenu. Elle révèle la faible attractivité économique des SCV proposés en raison du surcroît de travail et d'intrants que ces nouveaux systèmes requièrent la première année de mise en ouvre. L'étude montre aussi que pour rendre les SCV plus attractifs, il serait nécessaire de combiner des adaptations de la technique, telles que la diminution des quantités de biomasse dans les paillis associée à un recours aux herbicides, avec des subventions. Des recherches complémentaires sont nécessaires pour mieux quantifier les services environnementaux qui justifieraient de telles subventions. Il est également nécessaire de comprendre les causes de variations spatiales et temporelles des performances agronomiques et économiques des SCV et des systèmes actuellement pratiqués. Enfin, il faudrait comparer les SCV à d'autres techniques de conservation des sols, dont en particulier la culture en terrasse qui se répand actuellement dans la région.
\end{abstract}

Mots clés : culture sous couvert végétal, exploitation agricole familiale, mulch, plante de couverture, Viet Nam

Thèmes : productions végétales, systèmes agraires, agronomie

\section{Abstract \\ Ecological intensification in the mountains of Vietnam: Constraints to the adoption of cropping systems based on mulches and cover crops}

Significant efforts are being pursued in the tropical world to develop and promote cropping systems based on mulch or cover crops (SCV, système de culture sous couverture végétale, cover crop systems), in order to reduce soil erosion and improve both nutrients and water balances. However, adoption of SCV by smallholders is often limited due to the important change induced by these techniques in the management of farm's resources. The objective of this study conducted in Vietnam was to assess the feasibility of SCV that had been previously elaborated at field scale for several farm types. The method was based on the simulation of rational households optimizing the use of their resources for food-security and income. We showed that the proposed SCV were not economically attractive because of the extra labour and inputs that these techniques require during the first years of implementation. We also showed that improving the attractiveness of those SCV would require both technical adjustments, such as the use of herbicides combined with the reduction of the amount of biomass in mulches, and subsidies. More research is needed in order to better quantify the environmental services that could legitimate such subsidies. It is also necessary to better understand the causes of spatial and temporal variations of the agronomic and economic performances of the SCV and of the currently 
practised cropping systems. Lastly, SCV should be compared to other soil conserving techniques, especially cultivation on dry terraces that is tends tending to spread out in the region.

Key words: cover plants, family farms, mulches, underplanting, Viet Nam

Subjects: agronomy, farming system, vegetal productions

$\mathrm{D}^{\prime}$

importants efforts sont menés dans le monde tropical pour mettre au point et promouvoir des systèmes de culture sous couverture végétale (SCV). Le principe général des SCV est la couverture plus ou moins complète du sol par un paillis organique mort ou vivant au moins pendant la période d'installation de la culture. La présence de ces paillis implique généralement une réduction, voire une suppression, du travail du sol. Une revue de la littérature (Erenstein, 2003) et une synthèse d'experts du Sud et du Nord (Lahmar et al., 2006) fournissent le bilan le plus récent des connaissances sur ces systèmes. Leur avantage immédiat le plus tangible, vérifié dans des écosystèmes variés, est qu'ils protègent efficacement les sols contre l'érosion. Le paillis modifie aussi l'ensemble de l'écologie de la parcelle cultivée. Il est généralement admis, bien que sur la base d'un nombre limité d'études, qu'à long terme s'accumulent des effets favorables de telle sorte que les rendements se stabilisent à un niveau élevé. C'est ce qui conduit à considérer les SCV comme une voie prometteuse d'intensification respectueuse de l'environnement, soit l'“ écointensification " (Cassman, 1999). Néanmoins le potentiel agroécologique des SCV ne se concrétise pas immédiatement, et des résultats parfois contradictoires ont été rapportés sur les performances des SCV à court terme, selon leurs modalités de mise en œuvre et les environnements.

Par ailleurs, l'adoption des SCV par les exploitations familiales de la zone intertropicale reste très limitée (Bolliger et al., 2006, Carsky et al., 2003; Erenstein, 2003; Lal, 2007). Une des principales explications de cet échec est, selon ces auteurs, que les SCV nécessitent de profondes transformations de l'utilisation des ressources en terre, main-d'œuvre, trésorerie, et capital dans l'économie d'exploitations ayant une marge de manœuvre déjà réduite pour ces facteurs. En outre, les agriculteurs pauvres sont générale- ment contraints à privilégier le court terme pour leurs décisions, de telle sorte que leur investissement dans la préservation des ressources naturelles n'est pas possible s'il ne procure pas un avantage immédiat, même lorsque les populations ont conscience des avantages à long terme (Barbier, 1998; Shiferaw et Holden, 1998).

Dans les régions montagneuses du nord du Vietnam, des recherches à l'échelle de parcelles cultivées ont été menées pour adapter des SCV à l'environnement local, dans le but de proposer des alternatives à des systèmes de culture conventionnels, jugés érosifs, pratiqués sur des versants pentus (Husson et al., 2001). L'objectif de la présente étude est d'évaluer la faisabilité des SCV pour les agriculteurs de ces régions compte tenu des contraintes de mobilisation des ressources des exploitations et des performances agronomiques à court terme des SCV proposés.

\section{Méthode}

La méthode présentée ici repose sur la modélisation d'exploitations agricoles en programmation mathématique de manière à simuler la décision d'agriculteurs devant choisir entre plusieurs solutions techniques pour satisfaire leurs objectifs économiques en fonction de contraintes de ressources. Les données permettant de construire le modèle et de paramétrer ces simulations proviennent d'essais agronomiques et d'enquêtes chez les producteurs dans deux sites complémentaires.

\section{Sites étudiés}

Le premier site, Ngoc Phai, dans le district de Cho Don et la région nord-est du Vietnam, correspond à l'implantation la plus ancienne (1998) d'un projet de recherche-action, le "projet Systèmes agraires de montagne" (SAM) dans le cadre duquel ont été mis au point les SCV étudiés. Ce site avait été choisi comme représentatif, pour les régions de montagne d'Asie du Sud-Est, à la fois des milieux naturels, de la pauvreté des populations, et des problèmes d'érosion que la mise en valeur des pentes faisait craindre dans le futur. Le second site, Na Son, district de Dien Bien Dong, dans la région nord-ouest du Vietnam, fait partie de la région d'intervention d'un projet de développement à la demande duquel le "projet SAM" avait plus récemment élaboré des propositions de SCV adaptées à l'environnement local. Ce site était complémentaire du précédent par les caractéristiques du climat, plus sec, et par son enclavement tel qu'une agriculture de subsistance s'y maintenait tandis que le boom économique du Vietnam avait favorisé l'intégration au marché de l'agriculture à Ngoc Phai et dans la plupart des autres zones montagneuses du nord du pays au début des années 2000.

Au moment de notre étude, à Ngoc Phai la principale culture annuelle sur pentes est le maïs, destiné à un élevage porcin dont la production est vendue. Le climat permet deux cycles par an pour le riz irrigué comme pour le maïs pluvial. Le climat plus sec de NaSon n'autorise qu'un seul cycle de riz ou de maïs pluvial par an. Les ménages y cherchent à satisfaire leurs besoins alimentaires avec la culture de riz pluvial sur les pentes, sauf pour les rares familles disposant de ressources en eau permettant la culture de riz irrigué sur terrasses. Le mais est cultivé soit pour compléter l'alimentation familiale si la production de riz est insuffisante, soit pour être valorisé par les petits élevages, soit encore pour être directement vendu.

Dans les deux sites, les sols des versants sont soit des sols acides ferralitiques développés sur roches cristallines soit des sols bruns plus fertiles développés sur calcaires. La conduite des cultures sur pentes est entièrement manuelle. 


\section{Modélisation et simulation des choix stratégiques d'activités}

Des typologies d'exploitations agricoles ont été établies à partir de travaux antérieurs (Castella et Quang, 2002 ; Erout et Castella, 2004). Sur cette base, des enquêtes détaillées sur une quinzaine d'exploitations dans chaque site ont été menées afin de connaître les différents facteurs de production et les stratégies des exploitations dans ces communes.

Nous avons retenu sept exploitations, représentant des cas contrastés du point de vue de la surface agricole par actif, de la répartition entre terres irrigables et terres de pente, et des rapports entre nombre d'actifs et nombre de dépendants (tableau 1).
Des modèles d'exploitation agricole ont été construits à l'aide de la programmation mathématique (Hazell et Norton, 1986). Ces modèles visent à reproduire le comportement de ménages qui auraient à sélectionner une combinaison d'activités (agricoles ou extra-agricoles) qui maximise le revenu du ménage, tout en assurant sa sécurité alimentaire et en respectant les diverses contraintes liées aux disponibilités des facteurs de production. Le modèle tient compte en particulier des exigences en travail et trésorerie des différentes activités productives possibles dans la région et des productions attendues de ces activités en fonction des différents milieux dans lesquels elles sont praticables. L'année agricole est divisée en trois périodes correspondant aux différentes saisons, de manière à imposer une répartition réaliste des flux de maind'œuvre et de trésorerie dans le temps. Avec cette méthode, l'adoption simulée d'une activité est vue comme une condition nécessaire, mais non suffisante, de son adoption dans la réalité (Jourdain, 1995 ; Flichman et Jourdain, 1998). L'horizon de simulation est d'une année, soit dans le cas des SCV la première année de leur mise en ouvre dans une parcelle. L'évolution à long terme du milieu sous l'influence des activités agricoles n'est donc pas prise en compte. Les coefficients techniques (performances, besoins en intrants, en temps de travail) des différentes activités possibles proviennent d'enquêtes approfondies auprès des exploitations retenues, sauf pour les SCV, dont les données proviennent d'essais agronomiques décrits dans la section

\section{Tableau 1. Principales caractéristiques des exploitations modélisées et résultats des simulations de la section " sensibilité de l'adoption à l'environnement économique ".}

Table 1. Main characteristics of the modelled farms and results of the simulations of section " sensibilité de l'adoption à l'environnement économique ".

\begin{tabular}{|c|c|c|c|c|c|c|c|c|}
\hline & \multirow{4}{*}{\begin{tabular}{l}
\multicolumn{1}{c}{ Exploitation } \\
\multicolumn{1}{c}{ Site } \\
Niveau d'accès \\
au marché
\end{tabular}} & NP1 & & NP3 & NS1 & NS2 & NS3 & NS4 \\
\hline & & \multicolumn{3}{|c|}{ Ngoc Phai } & \multicolumn{4}{|c|}{ Na Son } \\
\hline & & \multicolumn{3}{|c|}{ Intégrée au marché } & \multicolumn{2}{|c|}{$\begin{array}{c}\text { En voie d'intégration } \\
\text { au marché }\end{array}$} & \multicolumn{2}{|c|}{$\begin{array}{c}\text { Agriculture } \\
\text { de subsistance }\end{array}$} \\
\hline & & $\begin{array}{l}\text { Grande } \\
\text { surface } \\
\text { en terres } \\
\text { irriguées }\end{array}$ & $\begin{array}{l}\text { Surface } \\
\text { en terres } \\
\text { irriguées } \\
\text { inter- } \\
\text { médiaire }\end{array}$ & $\begin{array}{l}\text { Faible surface } \\
\text { en terres } \\
\text { irriguées et } \\
\text { main-d'œuvre } \\
\text { abondante } \\
\end{array}$ & $\begin{array}{l}\text { Avec terres } \\
\text { irriguées }\end{array}$ & $\begin{array}{l}\text { Sans terres } \\
\text { irriguées, } \\
\text { main- } \\
\text { d'œuvre } \\
\text { abondante }\end{array}$ & $\begin{array}{l}\text { Fortement } \\
\text { dépendante } \\
\text { du riz pluvial }\end{array}$ & $\begin{array}{l}\text { Fortement } \\
\text { contrainte } \\
\text { en terre }\end{array}$ \\
\hline \multirow[t]{5}{*}{$\begin{array}{l}\text { Surface } \\
\left(\mathrm{m}^{2}\right)\end{array}$} & $\begin{array}{l}\text { Terres irriguées } \\
\text { de bas-fonds }\end{array}$ & 4900 & 1500 & 700 & 0 & 0 & 0 & 0 \\
\hline & $\begin{array}{l}\text { Terrasses } \\
\text { irriguées }\end{array}$ & 0 & 0 & 1700 & 3200 & 0 & 0 & 0 \\
\hline & $\begin{array}{l}\text { Sols bruns } \\
\text { fertiles } \\
\text { sur versants }\end{array}$ & 3800 & 3000 & 0 & 15100 & 58200 & 12300 & 0 \\
\hline & $\begin{array}{l}\text { Sols } \\
\text { ferralitiques } \\
\text { et autres sols } \\
\text { pauvres } \\
\text { sur versant }\end{array}$ & 0 & 2000 & 44700 & 9600 & 16900 & 19900 & 30300 \\
\hline & $\begin{array}{l}\text { Sols de } \\
\text { terrasses } \\
\text { alluviales } \\
\text { non irriguées }\end{array}$ & 0 & 0 & 400 & 0 & 0 & 0 & 0 \\
\hline \multirow[t]{2}{*}{ Nombre } & Dépendants & 5,0 & 5,0 & 6,0 & 8,0 & 12,0 & 6,0 & 7,0 \\
\hline & Actifs & 2,0 & 2,3 & 4,1 & 2,8 & 7,0 & 3,0 & 3,0 \\
\hline \multicolumn{2}{|c|}{$\begin{array}{l}\text { Subvention nécessaire } \\
\text { pour provoquer l'adoption } \\
\text { complète des SCV } \\
\text { dans les simulations } \\
\text { (USD/ha) }\end{array}$} & 188 & 125 & 125 & 93 & 131 & 154 & 174 \\
\hline
\end{tabular}

SCV : système sous couverture végétale ; USD : dollars US. 
suivante. Les prix ont été obtenus par enquête auprès des commerçants et services agricoles locaux.

Dans un premier temps, les SCV n'ont pas été intégrés comme activités potentielles dans les modèles, afin de calibrer ces derniers et de s'assurer qu'ils restituaient le plus fidèlement possible la réalité observée en termes d'activités d'élevage, de production végétale, et d'activités extraagricoles pour chaque type de ménage.

Des simulations ont ensuite été réalisées en intégrant les SCV comme activités potentielles de manière à tester leur adoption par les exploitations simulées. Enfin, une seconde série de simulations a été produite pour explorer l'impact d'adaptations techniques ou de changements de l'environnement économique (prix des intrants, subventions) sur l'adoption des SCV par les agriculteurs simulés.

\section{Essais agronomiques}

Les données de rendements et de besoins en travail et en intrants relatifs aux SCV et nécessaires aux simulations du modèle de décision proviennent de dispositifs agronomiques présents sur chaque site dans des parcelles louées à des agriculteurs. Un ensemble de SCV sur riz pluvial et maïs y était comparé à des systèmes de culture avec travail du sol et sans mulch, dans le but de fournir aux agriculteurs de chacun des sites une démonstration des performances et caractéristiques des SCV qui avaient été préalablement mis au point localement (Chabanne, comm. pers.). Les données de chaque essai peuvent donc être assimilées à l'information dont les producteurs disposent "au mieux", sur chaque site, pour choisir entre les systèmes de culture actuels et les SCV proposés. Les essais comportaient quatre répétitions de parcelles élémentaires de $100 \mathrm{~m}^{2}$. Les temps de travaux nécessaires à chaque opération culturale ont été évalués sur la somme des quatre répétitions (soit $400 \mathrm{~m}^{2}$ ). Les rendements ont été mesurés sur des placettes de $16 \mathrm{~m}^{2}$ (maiis) ou $4 \mathrm{~m}^{2}$ (riz) à raison de deux placettes par parcelle élémentaire. Les SCV avaient été mis au point avec un parti pris d'éviter autant que possible les herbicides, jugés peu compatibles avec l'économie de subsistance qui prévalait au début du "projet SAM " dans toute la région. En conséquence, une préparation sommaire de la parcelle était nécessaire, en plus de l'épandage du paillis, pour éliminer les mauvaises herbes les plus envahissantes. En outre, les quantités de paillis à épandre avaient été établies pour garantir leur efficacité à réduire la croissance des adventices, et la biomasse des résidus de culture du cycle précédent étant insuffisante pour cela, s'y ajoutait une biomasse collectée dans le voisinage des parcelles cultivées. Des systèmes avec mulch simple, SCV1, et des systèmes avec mulch et plante de couverture, SCV2, sont pris en compte. La plante de couverture des SCV2 a pour but de contribuer à la biomasse nécessaire à l'élaboration des mulchs, et de fournir un éventuel surplus de biomasse valorisable comme fourrage.

La présente étude n'a retenu, parmi les nombreux SCV testés dans ces essais, que ceux dont les marges brutes à l'hectare et par journée de travail ne sont pas toutes deux significativement inférieures à celles des témoins. Les SCV ainsi considérés sont décrits au tableau 2.

\section{Tableau 2. Systèmes de culture étudiés.}

Table 2. Cropping systems studied.

\begin{tabular}{|c|c|c|c|c|c|c|c|}
\hline Site & \multicolumn{3}{|c|}{ Ngoc Phai } & \multicolumn{4}{|c|}{ Na Son } \\
\hline $\begin{array}{l}\text { Nombre de cycles } \\
\text { par an }\end{array}$ & \multicolumn{3}{|l|}{2} & \multicolumn{4}{|l|}{1} \\
\hline Culture principale & \multirow{2}{*}{\multicolumn{2}{|c|}{$\begin{array}{l}\text { Maïs } \\
\text { Conventionnel SCV1 }\end{array}$}} & & \multirow{2}{*}{\multicolumn{2}{|c|}{$\begin{array}{c}\text { Maïs } \\
\text { Conventionnel SCV1 }\end{array}$}} & \multirow{2}{*}{\multicolumn{2}{|c|}{$\begin{array}{c}\text { Riz pluvial } \\
\text { Conventionnel SCV1 }\end{array}$}} \\
\hline Système de culture & & & $\mathrm{SCV} 2$ & & & & \\
\hline Préparation du sol & $\begin{array}{l}\text { Labour } \\
\text { à la houe }\end{array}$ & $\begin{array}{l}\text { Travail } \\
\text { superficiel } \\
\text { (sarclage } \\
\text { à la houe) }\end{array}$ & $\begin{array}{l}\text { Travail } \\
\text { superficiel } \\
\text { (sarclage } \\
\text { à la houe) }\end{array}$ & \begin{tabular}{|l|} 
Labour \\
à la houe
\end{tabular} & $\begin{array}{l}\text { Travail } \\
\text { superficiel } \\
\text { (sarclage } \\
\text { à la houe) }\end{array}$ & $\begin{array}{l}\text { Labour à la } \\
\text { houe }\end{array}$ & $\begin{array}{l}\text { Travail } \\
\text { superficiel } \\
\text { (sarclage } \\
\text { à la houe) }\end{array}$ \\
\hline Paillis & $\begin{array}{l}\text { Non, résidus } \\
\text { brûlés }\end{array}$ & $\begin{array}{l}\text { Oui, } \\
7 \text { t MS/ha }\end{array}$ & $\begin{array}{l}\text { Oui, } \\
7 \text { t MS/ha }\end{array}$ & $\begin{array}{l}\text { Non, résidus } \\
\text { brûlés }\end{array}$ & $\begin{array}{l}\text { Oui, } \\
7 \text { t MS/ha }\end{array}$ & $\begin{array}{l}\text { Non, résidus } \\
\text { brulés }\end{array}$ & $\begin{array}{l}\text { Oui, } \\
7 \text { t MS/ha }\end{array}$ \\
\hline $\begin{array}{l}\text { Semis culture } \\
\text { principale }\end{array}$ & En poquets & En poquets & En poquets & En poquets & En poquets & À la volée & En poquets \\
\hline Traitements semences & Non & $\begin{array}{l}\text { Fongicide }+ \\
\text { insecticide }\end{array}$ & $\begin{array}{l}\text { Fongicide }+ \\
\text { insecticide }\end{array}$ & Non & $\begin{array}{l}\text { Fongicide + } \\
\text { insecticide }\end{array}$ & Non & $\begin{array}{l}\text { Fongicide + } \\
\text { insecticide }\end{array}$ \\
\hline Plante de couverture & Non & Non & $\begin{array}{l}\text { Oui, Mucuna } \\
\text { pruriens } \\
\text { semé à } \\
45 \text { jours pour } \\
\text { chaque cycle, } \\
\text { fauché à la } \\
\text { fin du } 1^{\text {er }} \\
\text { cycle. }\end{array}$ & Non & Non & Non & Non \\
\hline Fertilisation minérale & $\begin{array}{l}\text { NPK 60-30- } \\
30 \text { unités/ha } \\
\text { en deux } \\
\text { apports }\end{array}$ & $\begin{array}{l}\text { NPK 90-70- } \\
60 \text { unités/ha } \\
\text { en deux } \\
\text { apports }^{a}\end{array}$ & $\begin{array}{l}\text { NPK 90-70- } \\
60 \text { unités/ha } \\
\text { en deux } \\
\text { apports }^{a}\end{array}$ & Non & $\begin{array}{l}\text { NPK 45-30- } \\
30 \text { unités/ha } \\
\text { en deux } \\
\text { apports }^{a}\end{array}$ & Non & $\begin{array}{l}\text { NPK 25-30- } \\
30 \text { unités/ha } \\
\text { en deux } \\
\text { apports }^{a}\end{array}$ \\
\hline
\end{tabular}

${ }^{a}$ : doses recommandées par les services locaux de vulgarisation agricole, plus un apport de 15 unités/ha de N au semis ; SCV : système sous couverture végétale ; MS : matière sèche. 


\section{Performances agronomiques et économiques des SCV testés en première ou seconde année de culture}

Seules les données de la première année de chaque essai ont été utilisées pour décrire les performances des SCV dans les simulations des exploitations. Néanmoins, ces essais ont été reconduits à l'identique l'année suivante et l'on dispose des données des deux premières années. Elles sont présentées ici de manière à vérifier l'absence d'un effet année trop marqué qui biaiserait nos simulations.

Dans l'essai de Na Son, le paillis augmente le rendement en grain de $45 \%$ pour le maïs, et de 18 à $31 \%$ selon les années pour le riz pluvial, par rapport au système conventionnel. Dans l'essai de Ngoc Phai, les deux SCV retenus dans l'étude n'améliorent les rendements qu'au cours du second cycle de culture en 2003, et le SCV2 améliore les rendements par rapport au système conventionnel et au SCV1, pour les deux cycles en 2004 (tableau 3). Aucune des différences entre traitements enregistrées à Ngoc Phai n'est statistiquement significative. En outre, ces essais n'ont pas été conçus de manière à permettre une analyse des causes des différences de rendement éventuellement enregistrées.

Les SCV retenus augmentent la productivité économique de la terre à $\mathrm{Na}$ Son mais non à Ngoc Phai, où les différences enregistrées ne sont pas significatives (figure 1A).

Â Na Son, les SCV testés ne modifient pas la productivité du travail. En revanche, à Ngoc Phai, ils engendrent une diminution significative de la productivité du travail de l'ordre de $30 \%$ (figure $1 \mathrm{~B}$ ). Cela provient surtout d'un surcroît de travail nécessaire pour la collecte de biomasse et son épandage dans les parcelles pour la constitution des paillis, non compensé par les réductions de temps de travail pour la lutte contre les adventices (figure 2). Les temps de semis sont également augmentés sous SCV du fait de la nécessité de ménager un espace libre de pailles au voisinage du poquet pour favoriser la levée, et, dans le cas du riz pluvial, de remplacer le semis à la volée par un semis en poquets.

Les données disponibles pour ces essais ne permettent pas d'interpréter les causes de variations des rendements entre traitements et entre les sites. Elles constituent un exemple de la variabilité des impacts des SCV sur les rendements et les temps de travaux selon les environnements mentionnés par Erenstein (2003) et Lahmar et al. (2006).

\section{Résultats}

\section{Simulations}

Peu ou pas d'adoption des SCV dans le contexte actuel

Après calage, les simulations réalisées sans inclure les SCV dans les activités possibles restituent correctement les portefeuilles d'activités observés des exploitations des deux sites.

Après introduction des SCV dans les modèles, aucun des agriculteurs simulés de Ngoc Phai n'adopte ces systèmes de culture. En revanche, les agriculteurs simulés de Na Son adoptent le maïs sur paillis et le riz pluvial sur paillis sur 10 à
$20 \%$ de la superficie totale de leurs exploitations. Les exploitations disposant à la fois de main-d'œuvre et de trésorerie (NS1, NS3 et NS4) choisissent préférentiellement les systèmes à base de maïs, tandis que le système à base de riz pluvial sur paillis n'est choisi que par l'exploitation NS2, qui dispose d'une maind'œuvre abondante et qui, contrairement aux autres, a davantage intérêt à maximiser la productivité de la terre que celle du travail. Sur les deux sites étudiés, ce sont les besoins supplémentaires en intrants (engrais, pesticides) mais surtout en travail pour la collecte et l'épandage du paillis qui limitent ou empêchent l'adoption des SCV par les producteurs simulés. Ces besoins en travail coïncident avec la période de préparation des sols pour le premier cycle de riz irrigué, fortement consommatrice de main-d'œuvre. Dans le cas de Ngoc Phai, le coût d'opportunité du travail est encore renchéri par le marché des activités extra-agricoles.

\section{Perspectives d'amélioration des techniques}

Compte tenu des résultats précédents, de nouvelles simulations ont été conduites pour explorer l'intérêt d'ajustements des SCV pour réduire leur exigence en temps de travail et/ou intrants. Les solutions simulées par le modèle ne sont pas notablement modifiées en considérant séparément :

- une réduction de $50 \%$ du temps de travail pour la collecte du paillis ;

- le déplacement de la date de collecte du paillis vers une période plus précoce de l'année et à plus faible coût d'opportunité du travail ;

- un coût nul des semences de Mucuna et un coût des fertilisants $\mathrm{P}$ et $\mathrm{K}$ au même

\section{Tableau 3. Résultats des essais agronomiques.}

Table 3. Results of the agronomic trials.

\begin{tabular}{|c|c|c|c|c|c|c|c|c|c|}
\hline \multirow[t]{2}{*}{ Culture } & \multirow[t]{2}{*}{ Site } & \multirow[t]{2}{*}{ Année } & \multirow[t]{2}{*}{ Cycle } & \multicolumn{2}{|c|}{ Conventionnel } & \multicolumn{2}{|c|}{ SCV1 (mulch) } & \multicolumn{2}{|c|}{ SCV2 (Mulch + Mucuna) } \\
\hline & & & & $\begin{array}{c}\text { Rendement } \\
\text { moyen } \\
(\mathrm{kg} / \mathrm{ha})\end{array}$ & Écart type & $\begin{array}{c}\text { Rendement } \\
\text { moyen } \\
(\mathrm{kg} / \mathrm{ha})\end{array}$ & Écart type & $\begin{array}{c}\text { Rendement } \\
\text { moyen } \\
(\mathrm{kg} / \mathrm{ha})\end{array}$ & Écart type \\
\hline \multirow{2}{*}{$\begin{array}{l}\text { Riz } \\
\text { pluvial }\end{array}$} & Na Son & 2004 & unique & 2309 & 798 & 2722 & 589 & & \\
\hline & & 2005 & unique & 2862 & - & 3750 & - & & \\
\hline \multirow[t]{5}{*}{ Maïs } & Na Son & 2004 & unique & 3410 & & 4935 & & & \\
\hline & Ngoc Phai & ai 2003 & 1 & 2665 & 1262 & 2076 & 887 & 2530 & 1263 \\
\hline & & 2003 & 2 & 1775 & 576 & 2285 & 891 & 2342 & 636 \\
\hline & & 2004 & 1 & 5207 & 1245 & 4823 & 1974 & 5701 & 1751 \\
\hline & & 2004 & 2 & 2298 & 1002 & 2301 & 1050 & 2644 & 2393 \\
\hline
\end{tabular}

SCV : système sous couverture végétale. 


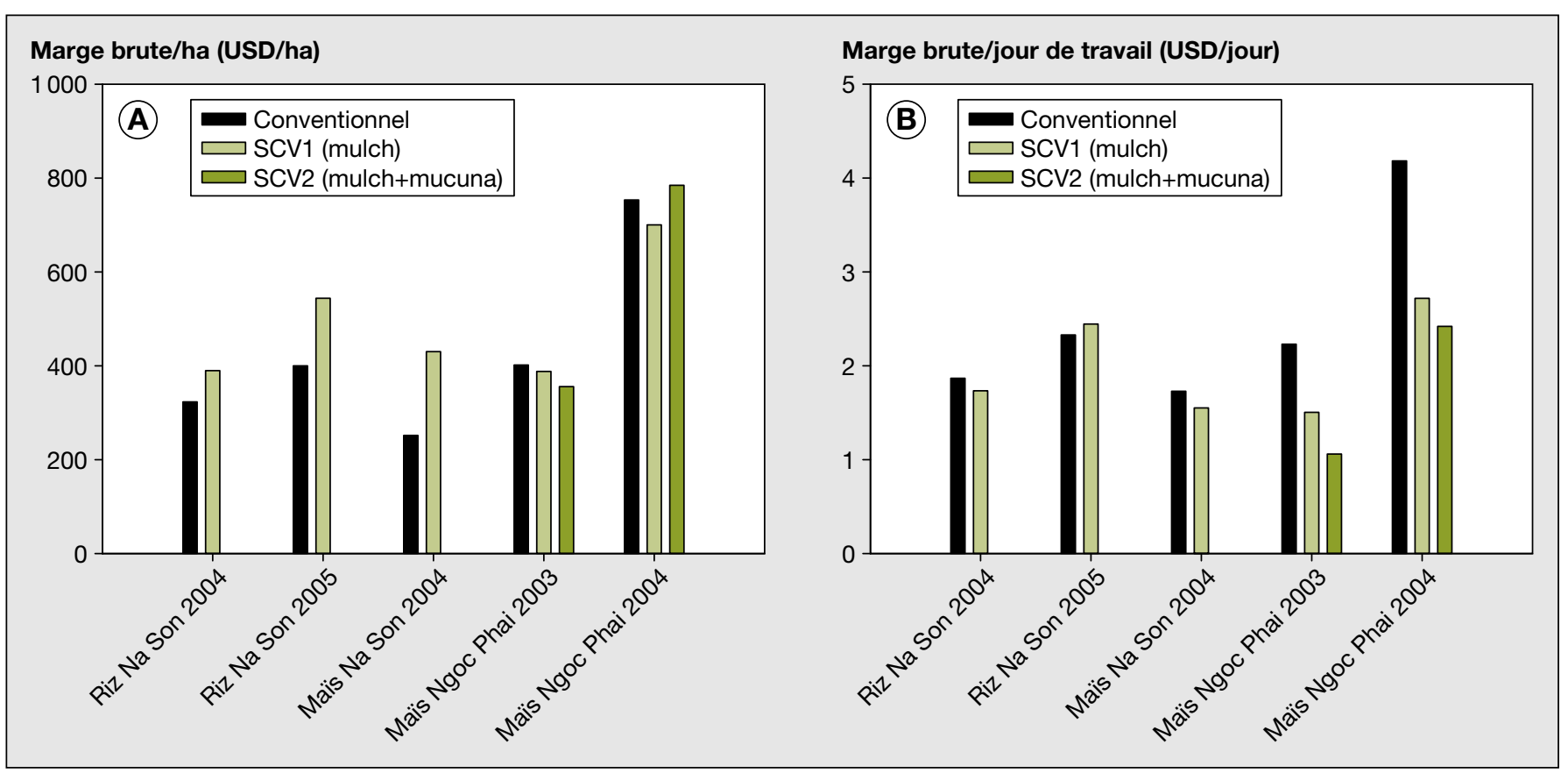

Figure 1. Performances économiques des systèmes de culture étudiés.

Figure 1. Economic performances of the studied cropping systems.

A) productivité de la terre ; B) productivité du travail.

SCV : système sous couverture végétale.

niveau que pour les systèmes de culture pratiqués par les agriculteurs.

Pour obtenir la simulation d'une adoption des SCV à Ngoc Phai, ou l'accroissement des surfaces cultivées en SCV à Na Son, il est nécessaire de combiner les réductions de travail et d'intrants évoquées ci-dessus.

\section{Sensibilité de l'adoption}

\section{à l'environnement économique}

Nous avons également simulé l'impact de primes octroyées aux producteurs pour l'adoption des SCV. Les primes nécessaires pour induire une adoption des SCV sur la totalité des surfaces de pentes des exploitations sont élevées par rapport aux marges brutes moyennes à l'hectare de tous les systèmes de culture (tableau 2, figure 1).

Par ailleurs, ce sont les systèmes SCV1 sur maïs qui sont adoptés dans les simulations, les niveaux de prime atteints n'étant pas suffisants pour induire l'adoption simulée du SCV2 (maïs-mucuna) à Ngoc Phai ou du riz sur paillis à Na Son.

Les fortes variations, entre exploitations, de ces primes calculées, témoignent de la variabilité des contraintes à l'adoption des SCV selon les exploitations.

\section{Discussion}

Les simulations ont mis en évidence des difficultés de mise en ouvre des SCV proposés dans les exploitations des montagnes du Vietnam principalement à cause de leur effet négatif ou nul sur la productivité du travail dans certains environnements, au moins lors de la première année de pratique. Ces difficultés sont sans doute à mettre en rapport avec le fait que, jusqu'à présent, les importants efforts de promotion des SCV par les services agricoles locaux auprès des agriculteurs de ces régions n'ont pas eu d'impact attesté. La nécessité, dans les simulations, de réduire simultanément les exigences en travail et en intrants nécessaires aux SCV pour provoquer leur adoption, laisse peu d'espoir quant à la réduction de ces difficultés par ajustements des techniques. Les experts ayant mis au point les SCV étudiés ici ont veillé à mobiliser la quantité minimale de mulch nécessaire pour assurer à la fois la lutte contre l'érosion, qui peut être efficace dès 1,5 à $2 \mathrm{t} /$ ha de matière sèche de pailles (Meyer et al., 1970 ; Smolikowski et al., 2007) et la lutte contre les adventices, qui a conduit à retenir $7 \mathrm{t} /$ ha de matière sèche de paille (Husson, comm. pers.). On peut donc s'attendre, et c'est également le discours des agriculteurs locaux avec qui cette question a été discutée, à ce qu'une réduction du temps de travail lié à la collecte du mulch ne soit envisageable qu'associée à un recours aux herbicides et donc à une augmentation des exigences en intrants.

Dans cette hypothèse, si l'on souhaitait favoriser l'adoption des SCV, il serait nécessaire de combiner un paillis à faible biomasse, le recours aux herbicides, et des subventions. Mais il faudrait au préalable analyser le coût d'une telle politique par rapport aux services environnementaux attendus. Si la lutte contre l'érosion peut sans doute être comptabilisée en service, la question de l'impact des herbicides sur l'environnement serait à considérer également.

Il faut relever ici que la culture en terrasse, actuellement promue avec un certain succès par les services agricoles vietnamiens, pourrait de ce point de vue présenter un avantage par rapport aux SCV. Elle permet évidemment une lutte efficace contre l'érosion. Mais elle facilite aussi la maitrise des mauvaises herbes, sans herbicides, car elle rend souvent possible la culture attelée, que les agri- 


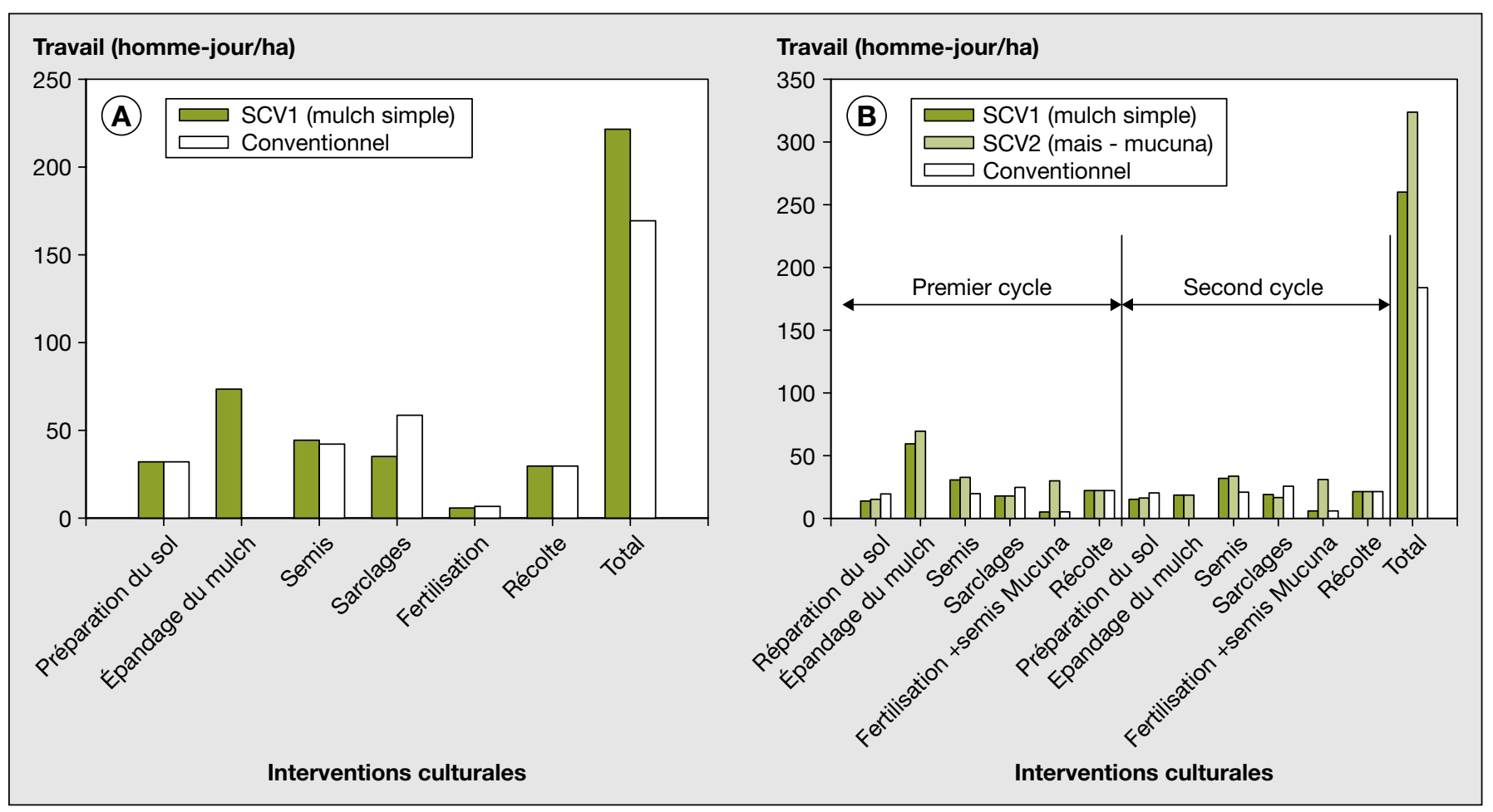

Figure 2. Temps de travail requis par les opérations culturales sous SCV et système de culture conventionnel.

Figure 2. Labour force required by crop management under SCV and conventional systems. A) sur riz pluvial dans l'essai de Na Son en 2004 ; B) sur maïs dans l'essai de Ngoc Phai en 2003. SCV : système sous couverture végétale.

culteurs pratiquent en général sur les terrasses alluviales des parties basses de leurs exploitations. Le temps de travail pour la construction de terrasses, voisin de 100 jours-homme/ha, est du même ordre que le temps de travail nécessaire à la collecte du paillis en première année d'installation de SCV.

Si l'on suppose une augmentation à long terme des productivités des SCV relativement aux systèmes conventionnels, la contrainte liée au temps de travail pourrait s'amenuiser avec le temps. Dans ce cadre, les faibles surfaces sur lesquelles les exploitants simulés de $\mathrm{Na}$ Son adoptent les SCV pourraient témoigner d'une possibilité d'expansion progressive de ces systèmes à mesure que les contraintes se réduisent sur les parcelles en SCV les plus anciennes. Cependant, nos simulations ne tiennent pas compte des contraintes éventuelles liées à la gestion des ressources en biomasse à l'échelle des territoires villageois, telles que des compétitions entre agriculteurs ou avec les activités pastorales pour l'accès à la biomasse nécessaire aux mulchs. Une analyse à cette échelle serait à conduire avant de promouvoir les SCV auprès des agriculteurs de cette zone.

Les difficultés repérées ici sont déjà évoquées dans les revues d'Erenstein (2003) et de Lahmar et al. (2006) comme pouvant se présenter dans certains systèmes agraires, mais sans qu'il soit proposé de démarche pour identifier ces cas. La méthode que nous avons utilisée nous paraît répondre à cette lacune pour ce qui concerne la faisabilité des SCV à l'échelle des exploitations, à condition de disposer d'un jeu de données permettant au moins de calculer les productivités de la terre et du travail pour les systèmes de culture comparés.

Les scénarios de simulation par lesquels nous avons exploré des modifications de la technique ou de l'environnement économique peuvent aussi être interprétés comme une analyse de la robustesse des solutions simulées vis-à-vis des paramètres explorés. De ce point de vue, on peut considérer que dans les sites étudiés, les difficultés identifiées sont bien réelles et ont peu de chances de résulter d'erreurs, même importantes, sur les temps de travaux et rendements associés aux systèmes conventionnels et aux SCV. Cepen- dant, il est possible que l'application de la méthode dans d'autres environnements ou types d'exploitation conduise à des solutions simulées beaucoup plus sensibles à la précision de ces paramètres, et qu'alors il soit nécessaire de recourir à des dispositifs agronomiques plus sophistiqués pour que l'étude soit concluante.

\section{Conclusion}

La modélisation des exploitations pour l'analyse des choix techniques des agriculteurs semble prometteuse pour étudier ex-ante les possibilités d'adoption des SCV par les agricultures familiales des pays en développement. Dans notre cas, elle a permis de montrer que les SCV testés sont loin de constituer une voie d'intensification écologique qui soit immédiatement diffusable dans les montagnes du Vietnam ou les régions similaires d'Asie du Sud-Est. Ils présentent en effet, au moins à court terme, des contraintes de temps de travail pour la collecte de biomasse, et dans une 
moindre mesure de trésorerie pour l'acquisition d'intrants, qui rendent économiquement difficile, pour la petite agriculture de subsistance mais plus encore pour une agriculture orientée vers le marché, leur intégration dans les systèmes de production actuels. Des ajustements techniques combinés à des primes, qui se justifieraient entre autres par la rémunération d'un service environnemental rendu à la société par les agriculteurs, permettraient d'améliorer les perspectives d'adoption de ces systèmes de culture. Préciser ces ajustements techniques et la légitimité d'une telle politique de subvention nécessiterait des travaux de recherche supplémentaires, notamment pour mieux connaître les causes des variations spatiales et temporelles des performances agronomiques des SCV et pour établir le bilan des services environnementaux rendus.

\section{Remerciements}

Ce travail a été réalisé dans le cadre du projet SAM (Systèmes agraires de montagne), coopération entre la Vietnam Academy of Agricultural Science (VAAS), l'International Rice Research Institute (IRRI) et le Centre de coopération internationale en recherche agronomique pour le développement (Cirad), avec un appui complémentaire du ministère français des Affaires étrangères. Les auteurs souhaitent remercier les agriculteurs des régions étudiées pour avoir trouvé le temps de participer à nos travaux d'enquêtes et nos expérimentations et pour leur accueil. Nos remerciements vont également à André Chabanne et Olivier Husson pour leur collaboration enthousiaste au début de cette étude. Nous espérons que les conclusions de ce travail leur seront utiles pour leur action en faveur de l'agroécologie.

\section{Références}

Barbier B. Induced innovation and land degradation : results from a bioeconomic model of a village in West Africa. Special issue: Food security, diversification, and resource management refocusing the role of agriculture? Agric Econ 1998 ; 19 : 15-25

Bolliger A, Magid J, Amado JCT, et al. Taking stock of the Brazilian "zero-till revolution": a review of landmark research and farmers" practice. Advances in Agronomy 2006; 91 : 47-110.

Carsky RJ, Douthwaite B, Manyong VM, et al. Lessons for appropriate soil management technology generation for the savannas and technology generation for the savannas and
their application to the grain legume-cereal their application to the grain legume-cere
rotation system. Cah Agric $2003 ; 12: 227-33$.

Cassman KG. Ecological intensification of cereal production systems : yield potential soil quality, and precision agriculture. National Academy of Sciences colloquium 1999; 96 : 5952-9.

Castella JC, Quang DD. Doi Moi in the Mountains. Land use hood strategies in Bac Kan Province, Viet Nam. Hanoi: The Agricultural Publishing House, 2002

Erenstein O. Smallholder conservation farming in the tropics and sub-tropics : a guide to the development and dissemination of mulching with crop residues and cover crops. Agric Ecosyst Environ 2003 ; 100 : 17-37.

Erout A, Castella JC. Lowland rice, upland rice : the cornerstones of agricultural production systems in a mountainous province of northern Vietnam. Cah Agric $2004 ; 13: 413-20$.
Flichman G, Jourdain D. Economic policy and water pollution. In : Wossink GAA, van Kooten GC, Peters GH, eds. Economics of agrochemicals. An international overview of use patterns, technical and institutional determinants, policies and perspectives. Wageningen (The Netherlands) : Ashgate, 1998.

Hazell PBR, Norton RD. Mathematical programming for economic analysis in agriculture. New York: Macmillan Publishing Company, 1986.

Husson O, Lienhard P, Seguy L, Tuan HD, Doanh LQ. Development of direct sowing and mulching techniques as alternatives to slashand-burn systems in northern Vietnam. Conservation agriculture, a worldwide chal lenge. First World Congress on conservation agriculture, Madrid, Spain, 1-5 October, 2001. Volume 2 : offered contributions, 2001.

Jourdain D. Utilisation des modèles bioéconomiques pour l'analyse des stratégies de protection des plantes : faisabilité, problèmes théoriques. DEA Economie du Développement Agricole, Agro-alimentaire et Rural. Montpellier : Ecole nationale supérieure agronomique lier : Ecole nationale supérieure
de Montpellier (Ensam), 1995.

Lahmar R, Arrúe JL, Denardin JE, Gupta RK, Ribeiro MFS, de Tourdonnet S. Knowledge assessment and sharing on sustainable agriculture. Main lessons. Montpellier : Centre de coopération internationale en recherche agronomique pour le développement (Cirad), 2006. http ://kassa.cirad.fr/results/kassa_main_results, 2006.

Lal R. Constraints to adopting no-till farming in developing countries. Soil Till Res 2007 ; 94 : 1-3.

Meyer LD, Wischmeier WH, Foster GR. Mulch rates required for erosion control on steep slopes. Sol Sci Soc Am Pro 1970 ; 34 : 928-30.

Shiferaw B, Holden ST. Resource degradation and adoption of land conservation technologies in the Ethiopian highlands: A case study in Andit Tid, North Shewa. Agric Econ 1998 ; $18: 233-47$.

Smolikowski $\mathrm{B}$, Puig $\mathrm{H}$, Roose $\mathrm{E}$. Influence of soil protection techniques on runoff, erosion and plant production on semi-arid hillsides of Cabo Verde. Agric Ecosyst Environ 2001; 87 : 67-80. 